\title{
Short-Term Outcomes of Atosiban in the Treatment of Preterm Labour at the Sultan Qaboos University Hospital, Muscat, Oman A tertiary care experience
}

"Nihal Al-Riyami, ${ }^{1}$ Hanin Al-Badri, ${ }^{2}$ Sanjay Jaju, ${ }^{3}$ Silja Pillai ${ }^{4}$

ABSTRACT: Objectives: This study aimed to generate baseline evidence regarding the effectiveness of atosiban in delaying delivery by $\geq 48$ hours among pregnant women presenting with threatened preterm labour (TPL). The secondary objective was to assess the relationship between atosiban success and various perinatal factors and neonatal outcomes. Methods: This retrospective study was conducted between June 2008 and May 2018 at the Sultan Qaboos University Hospital, Muscat, Oman. The medical records of all pregnant women who received atosiban between 24-34 gestational weeks for TPL during this period were reviewed. Results: A total of 159 women were included in the study. Atosiban was successful in delaying delivery by $\geq 48$ hours in 130 cases (81.8\%). Approximately half of the women ( $50.9 \%$ ) achieved uterine quiescence in $<12$ hours. Failure to delay delivery by $\geq 48$ hours was significantly lower among women with normal versus abnormal cervical findings $(11.1 \%$ versus $25.6 \%$; $P=0.023)$. Only $9.4 \%$ of women experienced minor side-effects. Mean birth weight $(2,724.55$ versus $1,707.59$ g; $P<0.001)$ and Apgar scores at 5 minutes $(9.66$ versus $8.28 ; P<0.001)$ were significantly higher among neonates delivered at $\geq 48$ versus $<48$ hours post-atosiban, whereas the rate of neonatal respiratory distress syndrome was significantly lower $(18.4 \%$ versus $81.6 \% ; P<0.001)$. Conclusion: Atosiban was highly effective in delaying delivery by $\geq 48$ hours and resulted in few adverse maternal side-effects and neonatal outcomes. To the best of the authors' knowledge, this is the first study conducted in Oman to evaluate the effectiveness of atosiban in preventing preterm labour.

Keywords: Preterm Labor; Atosiban; Tocolytic Agents; Treatment Outcome; Patient Outcome Assessment; Oman.

\section{AdvanCes IN KNOWLedge}

The results of this study will inform future research comparing the effectiveness and safety profile of atosiban to other tocolytic agents such as nifedipine.

To the best of the authors' knowledge, this is the first study conducted in Oman to evaluate the use of atosiban as a tocolytic drug for managing threatened preterm labour (TPL).

Application to Patient Care

The findings of this study indicate that atosiban is highly effective in delaying delivery by $\geq 48$ hours among selected patients presenting with TPL. In addition, the successful administration of this agent resulted in few maternal side-effects or adverse neonatal outcomes.

$\mathrm{P}$ RETERM BIRTH (PTB) IS A LEADING CAUSE of perinatal morbidity and mortality worldwide. ${ }^{1}$ Threatened preterm labour (TPL) is defined as regular uterine contractions leading to cervical changes before 37 gestational weeks that may result in PTB. $^{2}$ Globally, the incidence of preterm labour has increased dramatically in recent years, mainly due to a rise in multiple pregnancies, use of assisted reproductive techniques and increase in birth rate among women over 34 years of age. ${ }^{3}$ In 2014, approximately $10.6 \%$ of live births were preterm, of which $84.7 \%$ occurred in the late preterm period (i.e. at 34-37 gestational weeks). ${ }^{4}$ The highest rate of PTB worldwide was reported in Asia and sub-Saharan Africa (81.1\%). ${ }^{4}$

Although the exact mechanisms behind preterm labour are still unclear, various risk factors have been identified such as multiple pregnancies, cervical disease, decidual haemorrhage, placental ischaemia, fetal endocrine activation, intrauterine infections and various immunological or allergic phenomena and inflammatory processes. ${ }^{2}$ Maternal ethnicity and a previous history of preterm delivery, undernutrition, smoking and stress may also contribute to preterm labour. ${ }^{5}$ In $30 \%$ of PTBs, preterm rupture of the membranes occurs at least one hour prior to the onset of uterine contractions, whereas $15-20 \%$ result from iatrogenic causes such as pre-eclampsia and fetal growth restriction which necessitate medically indicated or elective preterm deliveries due to maternal or fetal indications. ${ }^{1,5}$ Late PTB occurs more often than early PTB and has significantly greater risks of adverse complications and outcomes compared to full-term births. ${ }^{4}$ 
Tocolytic drugs are the primary form of pharmacological management for TPL and are used to inhibit uterine contractions in a current episode of preterm labour as a first-line therapy or to maintain uterine relaxation after an acute episode as maintenance therapy. ${ }^{6,7}$ Tocolytic agents aim to prolong pregnancy by at least 48 hours so that pregnant women with TPL can be transferred to a tertiary care centre and fetal lung maturation can be induced with glucocorticoids. Tocolytic drugs include $\beta$-adrenergic receptor agonists, oxytocin receptor antagonists, calcium channel blockers, nitric oxide, prostaglandin synthase inhibitors and magnesium sulphate. ${ }^{8}$

Atosiban is a synthesised cyclic nonapeptide that behaves as a competitive oxytocin receptor antagonist to inhibit the oxytocin-mediated increase of intracellular calcium concentration that induces uterine muscle contractions. ${ }^{9,10}$ In the late 1980s, the first pilot study of atosiban analysed its effect on 13 patients and demonstrated its dose-dependent tocolytic effect. ${ }^{11}$ It is administered as an initial bolus of $6.75 \mathrm{mg}$ over the first minute followed by an infusion of $18 \mathrm{mg}$ /hour for three hours, before being reduced to $6 \mathrm{mg}$ /hour for up to 45 hours..$^{5}$ A recent meta-analysis found that atosiban was as effective as betamimetics in prolonging pregnancy and neonatal development. ${ }^{12}$ Maternal side-effects are rare $(<1 \%)$ and may include nausea, headaches and vomiting. ${ }^{12,13}$ A follow-up study showed that the drug had no adverse effects on neonatal motor and psychosocial development up to the age of two years. ${ }^{8}$ However, a more recent study showed some behavioural effects between 2.5 and 5.5 years. ${ }^{14}$

In Oman, atosiban is the most common tocolytic agent administered to pregnant women presenting with TPL. However, there is a lack of data regarding the effectiveness of atosiban for this purpose and its impact on maternal and perinatal outcomes. This study was therefore conducted to generate baseline evidence regarding the effectiveness of atosiban in preventing preterm labour and to establish the proportion of women who achieved complete uterine quiescence at 48 hours. In addition, the study aimed to examine the relationship between atosiban success and neonatal outcomes and degree of cervical dilatation at admission, as well as to document the occurrence of maternal side-effects.

\section{Methods}

This retrospective study was conducted from June 2008 to May 2018 at the Sultan Qaboos University Hospital (SQUH), a tertiary care institution in Muscat, Oman. All pregnant women between 24-34 gestational weeks who presented with TPL and received atosiban during this period were included in the study. A diagnosis of TPL was based on the presence of regular uterine contractions-defined as $2-3$ contractions of $\geq 30$ seconds' duration every 30 minutes-confirmed by external tocography, alongside evidence of cervical dilatation of $0-3 \mathrm{~cm}$ in nulliparous women or $1-3$ $\mathrm{cm}$ in primi- or multiparous women with $\geq 50 \%$ effacement. Women with multiple pregnancies, ruptured membranes, chorioamnionitis, severe preeclampsia, antepartum haemorrhage or allergy to atosiban were excluded from the study, as were those with less frequent contractions or no cervical changes.

Data were collected from the patients' electronic medical records on the hospital information system including maternal clinical and demographic characteristics (i.e. maternal age, gravidity, parity, gestational age at both admission and delivery, time to achieve uterine quiescence and cervical length and dilatation at admission). In addition, any adverse maternal effects from atosiban use were recorded such as headaches, vertigo, hypotension or tachycardia. Subsequently, information regarding neonatal outcomes was also gathered included birth weight, Apgar score, admission to the neonatal intensive care unit (NICU) and complications such as respiratory distress syndrome (RDS), necrotising enterocolitis and hyperbilirubinaemia. Following delivery, all neonates received at least one dose of $12 \mathrm{mg}$ of dexamethasone.

For the purposes of the study, atosiban success was defined as delivery delaying by $\geq 48$ hours. In order to assess the relationship between degree of cervical dilatation at admission and atosiban success, patients were divided into two groups. The first group consisted of women with normal cervical findings in whom the cervix was dilated to $0-10 \mathrm{~mm}$ in nulliparous or $10-15 \mathrm{~mm}$ in primi- or multiparous women; the second group was composed of women with abnormal cervical findings in whom the cervix was dilated to $>10 \mathrm{~mm}$ in nulliparous or $>15 \mathrm{~mm}$ in primi- or multiparous women.

Data were analysed using the Statistical Package for the Social Sciences (SPSS), Version 23.0 (IBM Corp., Armonk, New York, USA). Continuous variables were tested for normality of distribution and presented as either means and standard deviations or medians, ranges and interquartile ranges (IQRs). Categorical variables were presented as frequencies and proportions. A Pearson's chi-squared test was used to examine differences between expected and observed frequencies for categorical variables. Neonatal outcomes such as birth weight and Apgar scores at 5 minutes were compared with regards to atosiban failure (i.e. delivery in $<48$ hours) or success (i.e. 
Table 1: Maternal characteristics of pregnant women presenting with threatened preterm labour to the Sultan Qaboos University Hospital, Muscat, Oman (N = 159)

$\begin{array}{lc}\text { Characteristic } & \text { Median (IQR; range) } \\ \text { Age in years } & 27(9 ; 16-40) \\ \text { Gravidity } & 2(3 ; 1-10) \\ \text { Parity } & 1(2 ; 0-7) \\ \text { Abortions } & 0(1 ; 0-6) \\ \begin{array}{l}\text { Gestational age at admission in } \\ \text { weeks }\end{array} & 31.4(3.5 ; 24-33.6) \\ \begin{array}{l}\text { Mean cervical dilatation at } \\ \text { admission in mm }(95 \% \mathrm{CI})\end{array} & 9.9(7.35-10.82) \\ \text { IQR = interquartile range; } C I=\text { confidence interval. }\end{array}$

delivery in $\geq 48$ hours). Differences were analysed using an independent-samples t-test or Mann-Whitney U test, as appropriate. A $P$ value of $<0.050$ was considered statistically significant.

Ethical approval for this study was obtained from the Medical Research \& Ethics Committee of the College of Medicine \& Health Sciences at Sultan Qaboos University (MREC\#1711).

\section{Results}

A total of 159 pregnant women received atosiban for TPL during the study period, of which 132 (83\%) subsequently delivered at SQUH. The median maternal age was 27 years (IQR: 9 years; range: $16-40$ years) and median gravidity, parity and abortions was 2 (IQR: 3 ; range: $1-10$ ), 1 (IQR: 2; range: $0-7$ ) and 0 (IQR: 1 ; range: 0-6), respectively. The median gestational age at admission was 31.4 weeks (IQR: 3.5 weeks; range: 24-33.6 weeks). Mean cervical dilatation at admission was $9.9 \mathrm{~mm}$ (95\% confidence interval: 7.35-10.82 $\mathrm{mm}$ ) [Table 1]. Based on degree of cervical dilatation, normal and abnormal cervical findings were reported in 81 (50.9\%) and 78 (49.1\%) women, respectively.

Atosiban was successful in delaying delivery by $\geq 48$ hours in 130 cases (81.8\%). Uterine quiescence was achieved in $<12$ hours in 81 women $(50.9 \%)$ and

Table 2: Time taken to achieve uterine quiescence among pregnant women presenting with threatened preterm labour to the Sultan Qaboos University Hospital, Muscat, Oman $(\mathrm{N}=159)$

\begin{tabular}{lc|} 
Time in hours & $\mathbf{n}(\%)$ \\
\hline Not achieved & $29(18.2)$ \\
$<12$ & $81(50.9)$ \\
$12-35.9$ & $47(29.6)$ \\
$36-48$ & $1(0.6)$ \\
$>48$ & $1(0.6)$
\end{tabular}

Table 3: Perinatal factors associated with atosiban success among pregnant women presenting with threatened preterm labour to the Sultan Qaboos University Hospital, Muscat, Oman (N = 159)

\begin{tabular}{|c|c|c|c|}
\hline \multirow[t]{2}{*}{ Factor } & \multicolumn{2}{|c|}{ n (\%) } & \multirow[t]{2}{*}{$P$ value $^{\dagger}$} \\
\hline & $\begin{array}{l}\text { Atosiban } \\
\text { success* } \\
(\mathrm{n}=130)\end{array}$ & $\begin{array}{c}\text { Atosiban } \\
\text { failure } \\
(\mathbf{n}=29)\end{array}$ & \\
\hline \multicolumn{3}{|c|}{ Gestational age in weeks } & 0.187 \\
\hline $24-27.6$ & $16(12.3)$ & $7(24.1)$ & \\
\hline $28-31.6$ & $56(43.1)$ & $13(44.8)$ & \\
\hline 32-33.6 & $58(44.6)$ & $9(31)$ & \\
\hline \multicolumn{3}{|c|}{ Cervical findings at admission } & 0.023 \\
\hline $\mathrm{Normal}^{\ddagger}$ & $72(55.4)$ & $9(31)$ & \\
\hline Abnormal & $58(44.6)$ & $20(69)$ & \\
\hline
\end{tabular}

*Defined as prolonging delivery by $\geq 48$ hours. ${ }^{+}$Calculated using a Chisquared test. ${ }^{\ddagger}$ Defined as cervical dilation of $0-10 \mathrm{~mm}$ in nulliparous or 10-15 mm in primi- or multiparous women.

12-36 hours in 47 women (29.6\%). However, uterine quiescence was only achieved in $>36$ hours for two women (1.3\%) and failed entirely in 29 women (18.2\%) [Table 2]. In terms of gestational age, atosiban was successful in 16 out of 23 women (69.6\%) at 24-27.6 gestational weeks compared to 56 out of 69 women $(81.2 \%)$ at $28-31.6$ weeks and 58 out of 67 women $(86.6 \%)$ at $32-33.6$ weeks $(P=0.187)$. The rate of atosiban failure was significantly lower among women with normal cervical findings at admission compared to those with abnormal findings (11.1\% versus $25.6 \%$; $P=0.023)$ [Table 3]. No maternal side-effects were reported in 144 women (90.6\%). The remaining 15 patients experienced minor side-effects, including hypotension (4.4\%), headaches (3.8\%), dizziness $(0.6 \%)$ or tachycardia $(0.6 \%)$.

Perinatal outcomes were analysed only for the 132 women who delivered at SQUH. The median gestational age at delivery was 37 weeks (IQR: 5.7

Table 4: Perinatal outcomes associated with atosiban success among pregnant women presenting with threatened preterm labour to and delivering at the Sultan Qaboos University Hospital, Muscat, Oman ( $\mathrm{N}=132)$

\begin{tabular}{|c|c|c|c|}
\hline \multirow[t]{2}{*}{ Outcome } & \multicolumn{2}{|c|}{ Mean \pm SD } & \multirow[t]{2}{*}{$P$ value $^{\dagger}$} \\
\hline & $\begin{array}{l}\text { Atosiban } \\
\text { success }^{*} \\
(n=103)\end{array}$ & $\begin{array}{c}\text { Atosiban } \\
\text { failure } \\
(n=29)\end{array}$ & \\
\hline $\begin{array}{l}\text { Birth } \\
\text { weight in } \\
\text { grams }\end{array}$ & $\begin{array}{c}2,724.55 \\
\pm \\
607.93\end{array}$ & $\begin{array}{c}1,707.59 \\
\pm \\
734.15\end{array}$ & $<0.001$ \\
\hline $\begin{array}{l}\text { Apgar } \\
\text { score at } 5 \\
\text { minutes }\end{array}$ & $\begin{array}{c}9.66 \\
\pm \\
0.75\end{array}$ & $\begin{array}{c}8.28 \\
\pm \\
2.09\end{array}$ & $<0.001$ \\
\hline
\end{tabular}


Table 5: Neonatal outcomes associated with atosiban success among pregnant women presenting with threatened preterm labour to and delivering at the Sultan Qaboos University Hospital, Muscat, Oman ( $\mathrm{N}=132)$

\begin{tabular}{|c|c|c|c|}
\hline \multirow[t]{2}{*}{ Outcome } & \multicolumn{2}{|c|}{ n (\%) } & \multirow[t]{2}{*}{$P$ value $^{\dagger}$} \\
\hline & $\begin{array}{l}\text { Atosiban } \\
\text { success* } \\
(\mathbf{n}=103)\end{array}$ & $\begin{array}{c}\text { Atosiban } \\
\text { failure } \\
(\mathbf{n}=29)\end{array}$ & \\
\hline \multicolumn{3}{|l|}{ RDS } & $<0.001$ \\
\hline Present & 19 (18.4) & $22(75.9)$ & \\
\hline Absent & $84(81.6)$ & $7(24.1)$ & \\
\hline \multicolumn{3}{|c|}{ Hyperbilirubinaemia } & $<0.001$ \\
\hline Present & $14(13.6)$ & $14(48.3)$ & \\
\hline Absent & 89 (86.4) & $15(51.7)$ & \\
\hline \multicolumn{3}{|c|}{ NICU admission } & $<0.001$ \\
\hline Yes & $28(27.2)$ & $27(93.1)$ & \\
\hline No & $75(72.8)$ & $2(6.9)$ & \\
\hline \multicolumn{3}{|c|}{ Neonatal death } & $<0.001$ \\
\hline Yes & $1(1)$ & $6(20.7)$ & \\
\hline No & $102(99)$ & $23(79.3)$ & \\
\hline
\end{tabular}

weeks; range: 24-41.3 weeks). Mean birth weight was significantly higher in cases in which atosiban was successful in delaying delivery by $\geq 48$ hours compared to those in which delivery occurred at $<48$ hours $(2,724.55 \pm 607.93$ g versus $1,707.59 \pm 734.15$ g; $P<0.001)$. In addition, the mean Apgar score at 5 minutes was significantly higher among neonates who were delivered $\geq 48$ hours after receiving atosiban compared to those delivered at $<48$ hours $(9.66 \pm 0.75$ versus $8.28 \pm 2.09 ; P<0.001$ ) [Table 4].

The frequency of neonatal RDS was significantly lower in neonates in whom atosiban was successful in delaying delivery by $\geq 48$ hours compared to those delivered at $<48$ hours $(18.4 \%$ versus $81.6 \%$; $P$ $<0.001)$. Atosiban success also resulted in a significant reduction in the rates of neonatal hyperbilirubinaemia (13.6\% versus 48.3\%; $P<0.001$ ) and NICU admission (27.2\% versus $93.1 \% ; P<0.001)$ compared to neonates in whom atosiban failed to prolong delivery by $\geq 48$ hours. Finally, there was a significant reduction in neonatal deaths among those in whom atosiban successfully delayed delivery by $\geq 48$ hours compared to those delivered at $<48$ hours (1\% versus $20.7 \%$; $P$ $<0.001$ ) [Table 5].

\section{Discussion}

In the current study, atosiban was found to be highly effective $(81.8 \%)$ in delaying delivery by $\geq 48$ hours among pregnant women presenting with TPL to a tertiary hospital in Oman. This finding agrees with previous studies showing a significant increase in the proportion of women in whom delivery was delayed by $\geq 48$ hours among those receiving atosiban compared to a placebo. ${ }^{15-18}$ Further analysis in the present study also showed that the success rate of atosiban increased alongside gestational age. The majority of women in the present study achieved uterine quiescence in $<12$ hours following the administration of atosiban. A similar study conducted in India showed that atosiban resulted in a statistically significant reduction in the frequency of uterine contractions. ${ }^{19}$

A large randomised trial of 531 women by Romero et al. concluded that the use of atosiban in patients with TPL in comparison to a placebo was effective in prolonging pregnancy by up to seven days for those at $\geq 28$ gestational weeks, with a low rate of maternal or fetal adverse effects. ${ }^{20}$ Similarly, the current study found that atosiban resulted in no serious adverse effects in terms of maternal safety profile, with only $9.4 \%$ of women reporting minor side-effects such as hypotension, headache, dizziness and tachycardia. Other studies have also reported fewer adverse effects with atosiban, with this agent appearing to be better tolerated by pregnant women compared to other tocolytic drugs. ${ }^{21-23}$

With regards to neonatal outcomes, the current study found that atosiban success was associated with a significant reduction in the frequency of RDS. This can be attributed to the fact that the administration of atosiban was successful in delaying delivery, thus allowing for the prolonged administration of corticosteroids to enhance fetal lung development. This is in agreement with the results of the present study, that showed statistical significance $(P<0.001)$. However, previous randomised trials comparing atosiban with other tocolytic drugs have shown that the former results in a higher rate of NICU admission. ${ }^{20,24}$ In contrast, the present study found that atosiban success was associated with a significant reduction in NICU admissions. This could be attributed to several factors including the careful selection of patients presenting with TPL and early intervention with atosiban, resulting in a high success rate in terms of delaying delivery by $\geq 48$ hours. In addition, most patients included in such trials are $<28$ gestational weeks, whereas the median gestational age in the current study was 31.4 weeks. ${ }^{25,26}$

According to previous research, the administration of atosiban has been found to result in a higher frequency of neonatal deaths compared to those who received a placebo. ${ }^{5,27}$ However, the present study observed a statistically significant reduction in neonatal deaths 
in cases in which atosiban was successful in delaying delivery by $\geq 48$ hours compared to neonates in whom delivery occurred at $<48$ hours. This could be attributed to the fact that other studies include a greater number of women at $<26$ gestational weeks randomised to the atosiban group, whereas most women in the present study were admitted at a more advanced gestational age. ${ }^{5,27}$

To the best of the authors' knowledge, this is the first study from Oman to evaluate the effectiveness of atosiban in prolonging pregnancy and preventing PTB among women with TPL. However, there were several limitations. In addition to the retrospective nature of the study design, the low sample size and inclusion of pregnant women from a single tertiary hospital could have affected the generalisation of the results. Moreover, neonatal outcome information was not available for those women who did not deliver at SQUH. Finally, it was not possible to compare outcomes among women receiving other tocolytic drugs, as atosiban has been the most commonly tocolytic agent used for the management of TPL in Oman over the past decade. Additional multicentre studies are therefore recommended to compare the effectiveness and safety profile of atosiban with other tocolytic agents.

\section{Conclusion}

The current study found atosiban to be highly effective in delaying delivery by $\geq 48$ hours in pregnant women with TPL, with very few adverse maternal side-effects or neonatal outcomes. However, further research is recommended to compare the safety and efficacy of atosiban with other cost-effective tocolytic drugs such as nifedipine.

\section{CONFLICT OF INTEREST}

The authors declare no conflicts of interest.

\section{FUNDING}

No funding was received for this study.

\section{References}

1. Beck S, Wojdyla D, Say L, Betran AP, Merialdi M, Requejo JH, et al. The worldwide incidence of preterm birth: A systematic review of maternal mortality and morbidity. Bull World Health Organ 2010; 88:31-8. https://doi.org/10.2471/BLT.08.062554.

2. Hubinont C, Debieve F. Prevention of preterm labour: 2011 update on tocolysis. J Pregnancy 2011; 2011:941057. https:// doi.org/10.1155/2011/941057.

3. Kinney MV, Lawn JE, Howson CP, Belizan J. 15 million preterm births annually: What has changed this year? Reprod Health 2012; 9:28. https://doi.org/10.1186/1742-4755-9-28.
4. Chawanpaiboon S, Vogel JP, Moller AB, Lumbiganon P, Petzold M, Hogan D, et al. Global, regional, and national estimates of levels of preterm birth in 2014: A systematic review and modelling analysis. Lancet Glob Health 2019; 7:e37-46. https://doi. org/10.1016/S2214-109X(18)30451-0.

5. Hamilton SA, Tower CL. Management of preterm labour. Obstet Gynaecol Reprod Med 2013; 23:114-20. https://doi. org/10.1016/j.ogrm.2013.02.009.

6. National Institute for Health and Care Excellence. Preterm labour and birth: NICE guideline [NG25]. From: www.nice. org.uk/guidance/ng25/documents/preterm-labour-and-birthdraft-guideline-nice2 Accessed: Jul 2020.

7. Berkman ND, Thorp JM Jr, Lohr KN, Carey TS, Hartmann KE, Gavin NI, et al. Tocolytic treatment for the management of preterm labor: A review of the evidence. Am J Obstet Gynecol 2003; 188:1648-59. https://doi.org/10.1067/mob.2003.356.

8. Schleußner E. The prevention, diagnosis and treatment of premature labor. Dtsch Arztebl Int 2013; 110:227-35. https:// doi.org/10.3238/arztebl.2013.0227.

9. Kim SH, Riaposova L, Ahmed H, Pohl O, Chollet A, Gotteland JP, et al. Oxytocin receptor antagonists, atosiban and nolasiban, inhibit prostaglandin F2 $\alpha$-induced contractions and inflammatory responses in human myometrium. Sci Rep 2019; 9:5792. https://doi.org/10.1038/s41598-019-42181-2.

10. Papatsonis DN, Flenady V, Liley HG. Maintenance therapy with oxytocin antagonists for inhibiting preterm birth after threatened preterm labour. Cochrane Database Syst Rev 2013; 10:CD005938. https://doi.org/10.1002/14651858.CD005938.pub3.

11. Papatsonis D, Flenady V, Cole S, Liley H. Oxytocin receptor antagonists for inhibiting preterm labour. Cochrane Database Syst Rev 2005; 3:CD004452. https://doi.org/10.1002/14651858. CD004452.pub2.

12. Flenady V, Reinebrant HE, Liley HG, Tambimuttu EG, Papatsonis DN. Oxytocin receptor antagonists for inhibiting preterm labour. Cochrane Database Syst Rev 2014; 6:CD004452. https://doi.org/10.1002/14651858.CD004452.pub3.

13. Lamont RF, Jørgensen JS. Safety and efficacy of tocolytics for the treatment of spontaneous preterm labour. Curr Pharm Des 2019; 25:577-92. https://doi.org/10.2174/13816128256661903 29124214.

14. van Winden TM, Klumper J, Kleinrouweler CE, Tichelaar MA, Naaktgeboren CA, Nijman TA, et al. Effects of tocolysis with nifedipine or atosiban on child outcome: Follow-up of the APOSTEL III trial. BJOG 2020; 127:1129-37. https://doi.org/10.111 1/1471-0528.16186.

15. de Heus R, Mulder EJ, Visser GH. Management of preterm labor: Atosiban or nifedipine? Int J Womens Health 2010; 2:137-42. https://doi.org/10.2147/IJWH.S7219.

16. Rundell K, Panchal B. Preterm labor: Prevention and management. Am Fam Physician 2017; 95:366-72.

17. Wathes DC, Borwick SC, Timmons PM, Leung ST, Thornton S. Oxytocin receptor expression in human term and preterm gestational tissues prior to and following the onset of labour. J Endocrinol 1999; 161:143-51. https://doi.org/10.1677/joe.0.16 10143.

18. Arrowsmith S, Kendrick A, Wray S. Drugs acting on the pregnant uterus. Obstet Gynaecol Reprod Med 2010; 20:241-7. https://doi.org/10.1016/j.ogrm.2010.05.001.

19. Dewan B, Shah D. The clinical experience of atosiban in preterm labour. J Adv Med Med Res 2016; 13:1-9. https://doi. org/10.9734/bjmmr/2016/23823.

20. Romero R, Sibai BM, Sanchez-Ramos L, Valenzuela GJ, Veille JC, Tabor B, et al. An oxytocin receptor antagonist (atosiban) in the treatment of preterm labor: A randomized, double-blind, placebocontrolled trial with tocolytic rescue. Am J Obstet Gynecol 2000; 182:1173-83. https://doi.org/10.1067/mob.2000.95834. 
21. Fullerton GM, Black M, Shetty A, Bhattacharya S. Atosiban in the management of preterm labour. Clin Med Insights Womens Health 2011; 4:9-16. https://doi.org/10.4137/cmwh.s5125.

22. Coomarasamy A, Knox EM, Gee H, Song F, Khan KS. Effectiveness of nifedipine versus atosiban for tocolysis in preterm labour: A meta-analysis with an indirect comparison of randomised trials. BJOG 2003; 110:1045-9. https://doi.org/10.1111/j.14710528.2003.03071.x.

23. Sanu O, Lamont RF. Critical appraisal and clinical utility of atosiban in the management of preterm labor. Ther Clin Risk Manag 2010; 6:191-9. https://doi.org/10.2147/tcrm.s9378.

24. Salim R, Garmi G, Nachum Z, Zafran N, Baram S, Shalev E. Nifedipine compared with atosiban for treating preterm labor: A randomized control trial. Obstet Gynecol 2012; 120:1323-31. https://doi.org/10.1097/AOG.0b013e3182755dff.
25. Tsatsaris V, Carbonne B, Cabrol D. Atosiban for preterm labour. Drugs 2004; 64:375-82. https://doi.org/10.2165/00003495200464040-00003.

26. Denny JM, Culhane JF, Goldenberg RL. Prevention of preterm birth. Womens Health (Lond) 2008; 4:625-38. https://doi. org/10.2217/17455057.4.6.625.

27. Meher S, Alfirevic Z. Choice of primary outcomes in randomized trials and systematic reviews evaluating interventions for preterm birth prevention: A systematic review. BJOG 2014; 121:1188-94. https://doi.org/10.1111/1471-0528.12593. 